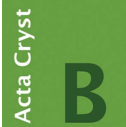

STRUCTURAL SCIENCE CRYSTAL ENGINEERING MATERIALS

ISSN 2052-5206

\section{Introduction to Nanofiber Materials. By Frank K. Ko and Yuqin Wan. Cambridge University Press, 2014. Pp. 282. Price GBP 65.00, US\$ 99.00. ISBN 978-0-521- 87983-5.}

\author{
Seeram Ramakrishnaa* \\ Center for Nanofibers and Nanotechnology, National University of Singapore, Create Research Wing, \# 03-08, 1 Create \\ Way, Singapore 138602, Singapore. *Correspondence e-mail: seeram@nus.edu.sg
}

'Introduction to Nanofiber Materials' is interesting as it is written for undergraduate and graduate students, as well as research scientists in material engineering, bioengineering and especially nanotechnology. The book is very well planned and well written, with a comprehensive introduction to nanosize scale materials and the chemistry of polymeric materials. An in-depth explanation of the basic properties of polymers helps the reader to refresh knowledge acquired during university courses, and is a very good introduction to further content, especially various methods of fiber formation, with understandable and clear schematics which are essential to fully understand nanofiber formation and their advantages and limits.

The chapter on nanofiber technology describes briefly methods of nanofiber production, with special focus on the electrospinning method, and emphasis placed on the influence of process parameters on fiber formation and diameter. Comprehensive knowledge about process parameters is essential when using the electrospinning method. Fiber formation by electrospinning depends on many various parameters, interacting together in many combinations. The work presented updates progress on understanding these dependencies and gives an explanation of the methodology of modelling and electrospinning simulation which still have not been fully understood. The authors of this work focus on the methods of characterization and test nanofibrous materials which have been divided very intuitively. The reader will learn about ways of characterizing polymeric materials with a number of useful examples of satisfying or unsatisfying results like spectra, visualizations and graphs.

The principles of every method described in the book are also explained in a very approachable way. Characterization methods are presented in accordance with recent research, which shows the reader how to apply those methods in the laboratory and how to interpret the results.

Electrospun polymeric fibers have been investigated extensively for the last decade. A large number of various polymeric materials were used to create nanofibrous sheets in various forms. In this work, the authors summarized the most recent research in bioactive nanofibers, electroactive fibers and nanocomposite fibrous materials. Fiber research has been extensively studied in recent years, and has a high potential for commercialization in the near future. In particular, the presented bioactive properties of nanofibers showed the methodology and possible future areas of development that researchers may be interested in. Nevertheless, the drug delivery section could be extended by including the electrospraying technique, which becomes a complementary method of creating polymeric drug delivery systems.

The electroactive properties of nanofibers result from their chemical composition and dimensions. The discovery of carbon nanotubes in 1991 revealed a huge potential for the production of electroconductive and supermagnetic materials. The simultaneous development of electrospinning and characterization techniques of nanomaterials brought a wide range of possible applications of these materials, which are described in-depth by the authors. The combination of various materials leads to the formation of a completely novel and promising group of nanocomposite fibrous textiles. Similar to previously mentioned components, authors emphasize the production of nanocomposites and their advantages and limitations, which may be a valuable guide for the experienced reader. 
The material reviewed lacks a list of commercially available products that could interest readers focused on entrepreneurism and commercialization. An attempt to predict the future of nanotechnology and commercialization of nanofibers is presented together with the characterization of different electrospinning set-ups and scaled up equipment.
I would recommend this book for students and researchers of various fields interested in the electrospinning process and nanotechnology research. This publication is valuable and comprehensive and will guide the reader through all steps of the creation, application and characterization of nanofiber materials. 